\title{
Nonradial linear oscillations of shells in general relativity
}

\author{
Bernd G. Schmidt* \\ Max-Planck-Institut für Gravitationsphysik, Albert-Einstein-Institut, Schlaatzweg 1, 14473 Potsdam, Germany
}

(Received 30 July 1998; published 7 December 1998)

\begin{abstract}
Nonradial perturbations of a static shell with an empty interior, or around a star or black hole, are investigated. The transition conditions at the shell are derived in a Gaussian and in Regge-Wheeler gauge. The existence of linearized shell solutions is demonstrated. Quasinormal modes are discussed, and the Newtonian limit of the equations is derived. [S0556-2821(98)04124-1]
\end{abstract}

PACS number(s): 04.20.Ex, 04.30.Db, 04.30.Nk

\section{INTRODUCTION}

Shells have been studied in Einstein theory in various contexts [1-7]. The existence of solutions which describe static, spherically symmetric shells around a black hole or with an empty interior having been established [3], one naturally asks for time-dependent linear perturbations of these solutions. Radial perturbations have been studied in [6]. As far I have been able to find out, nonradial perturbations have never been treated. Recently [7], nonradial perturbations of a bubble which separates a Minkowski from a de Sitter region were analyzed.

A treatment of nonradial oscillations should be quite straightforward. One just has to take linearized vacuum solutions inside and outside the background shell and to impose certain transition conditions which contain information on the perturbation of the shell. Because of gauge problems, this turned out to be more complicated than I expected. It is tempting to speculate that all this should become much simpler if one uses Moncrief's gauge-invariant formulation and wave equation. I found, however, no way of expressing the fluid shell transition conditions directly in a gauge-invariant variable. The reason is probably that the second fundamental forms defining the stress-energy tensor of the shell contain always gauge-dependent parts of the metric.

My motivation to study this problem came from the hope that certain questions which turn up in the linearized theory of stellar oscillations [8] could be treated more easily if the oscillating star were replaced by an oscillating shell. The questions I want to study are related to the Newtonian limit: normal modes versus quasinormal modes, and do oneparameter families of relativistic oscillating shells exist which have a Newtonian limit?

The paper is organized as follows. Section II describes shells in the "frame theory" [9], a theory which contains a parameter $\lambda$ such that $\lambda>0$ gives Einstein's theory and $\lambda$ $=0$ Newton's. This formulation turns out to be very useful in obtaining the Newtonian limit. Section III deals with the linearization and derives the transition conditions in a Gaussian gauge. To deal later with questions of existence, the transition conditions are also calculated in a Regge-Wheeler gauge where they are very lengthy.

In Sec. IV the existence of linearized shell solutions is

*Email address: bernd@aei-potsdam.mpg.de demonstrated by the method of characteristics as was done in [8] in the case of stellar oscillations. It is shown that the Cauchy problem is well posed. An excitation of the gravitational field outside the shell sets the shell in motion, which in turn will then be damped by the radiation it emits. Conversely, a displacement of the shell from its equilibrium position will induce a motion of the shell and radiation. This is the first time that a Cauchy problem is posed and solved for shells in Einstein's theory, unfortunately only in a linearized version.

Section V discusses quasinormal modes. It is shown how the transition conditions at the shell which are derived in Sec. III can be used to calculate numerically the quasinormal mode frequencies of the shell.

Section VI deals with the Newtonian limit. The ordinary differential equations describing linearized self-gravitating Newtonian shells are derived as limits from the relativistic equations. This limit process is rather subtle, because the Gaussian gauge, which is natural in the relativistic picture, is not so natural for the usual formulation of Newton's theory in an inertial frame. Nevertheless, the correct Newtonian equations can be found as a limit from Einstein's equations and the transition conditions at the shell.

\section{SHELLS IN THE FRAME THEORY}

The "frame theory" formulated by Ehlers [9] is a theory containing a parameter $\lambda$ such that for $\lambda=0$ we have Newton-Cartan-Friedrichs theory in general, Newton's if $R^{\alpha \beta} \cdot \gamma \delta=0$, in particular if spatially asymptotically flat, and for $\lambda>0$ Einstein's theory of gravitation. The basic variables are the time metric $t_{\alpha \beta}$, the space metric $s^{\alpha \beta}$, which satisfy $t_{\alpha \beta} s^{\beta \gamma}=-\lambda \delta_{\alpha}^{\gamma}$, and, most important, a symmetric linear connection $\Gamma_{\alpha \beta}^{\gamma}$, which has the property that both metrics are covariantly constant. Matter is described by a tensor $T^{\alpha \beta}$. The field equations are written as

$$
R_{\alpha \beta}=8 \pi\left(T_{\alpha \beta}^{\cdot \bullet}-\frac{1}{2} t_{\alpha \beta} T_{\delta}^{\delta_{\delta}}\right) \quad(G=1)
$$

The indicates where the index was before it was raised or lowered with $s^{\alpha \beta}$ or $t_{\alpha \beta}$. The equations of motion, $T_{; \beta}^{\alpha \beta}$, have to be postulated independently, because in Newtonian theory they do not follow from the field equations.

To describe shells in the frame theory, we need the concept of a second-fundamental form for the Newtonian con- 
nection. If $n_{\alpha}$ is a one-form normal to a timelike hypersurface $\Sigma$, we can normalize it by the space metric such that $n_{\alpha} n_{\beta} s^{\alpha \beta}=1$. The vector field $n_{\text {. }}^{\alpha}$ is transversal to $\Sigma$ and, in the case $\lambda=0$, orthogonal to $\Sigma_{t}$, the intersection of $\Sigma$ with the Newtonian time slices $t=$ const.

Using this vector field $n_{\bullet}^{\alpha}$, we can define a "secondfundamental" form $k_{\mu \nu}$ of $\Sigma$ which is equivalent to the usual one for $\lambda \neq 0$ : Let $\xi^{\alpha}$ and $\eta^{\beta}$ be two vector fields tangent to $\Sigma$ and decompose $\xi_{; \beta}^{\alpha} \eta^{\beta}$ into its components tangential to $\Sigma$ and in the direction of $n_{\text {. }}^{\alpha}$ :

$$
\xi_{; \beta}^{\alpha} \eta^{\beta}=\xi_{\mid \beta}^{\alpha} \eta^{\beta}+k_{\mu \nu} \xi^{\mu} \eta^{\nu} n_{\bullet}^{\alpha}, \quad k_{\mu \nu} n_{\bullet}^{\nu}=0,
$$

to define the second-fundamental form as usual. It can be checked that " "|, defines a symmetric connection on $\Sigma$ and that $k_{\mu \nu}$ is symmetric.

In general relativity shells have been defined and discussed, for example, by Israel [1]. We proceed similarly in the frame theory.

A shell is a timelike hypersurface $\Sigma$ in a manifold with continuous time and space metrics and a connection, with the following properties: (1) the metrics and the connection are smooth on the complement of $\Sigma$ and satisfy all axioms of the frame theory in empty space, in particular, $R_{\alpha \beta}=0$ [9]; (2) (in some coordinate system) partial derivatives of the metrics and the connection of any order have limits from both sides at $\Sigma$.

These conditions imply that the induced space and time metrics on $\Sigma$ coincide and are smooth. The secondfundamental forms ${ }^{ \pm} k_{\alpha \beta}$ from both sides exist. In the Newtonian case the components of the second-fundamental form in the $t=$ const time hypersurfaces are continuous.

The Ricci tensor is distributional at the shell:

$$
R_{\mu \nu} \Pi_{\alpha}^{\mu} \Pi_{\beta}^{\nu}=8 \pi \delta_{\Sigma} \Delta k_{\alpha \beta},
$$

where $\Pi_{\alpha}^{\mu}=\delta_{\alpha}^{\mu}-n_{\bullet}^{\mu} n_{\alpha}$ projects onto $\Sigma$. Here $\delta_{\Sigma}$ is a distribution which has support on $\Sigma$ and could be defined using, for example, Gauss coordinates relative to $\Sigma$. For $\lambda>0$ this follows from relations which express four-dimensional quantities in terms of three-dimensional ones and the secondfundamental form. In the case $\lambda=0$ it can be checked in coordinates adapted to the Newtonian case.

The field equations (2.1) and the relation (2.3) motivate the definition of the energy-momentum tensor of the shell $T^{\alpha \beta}=\delta_{\Sigma} \tau^{\alpha \beta}$, which is related to the jump of the secondfundamental form by

$$
\Delta k_{\alpha \beta}={ }^{+} k_{\alpha \beta}-{ }^{-} k_{\alpha \beta}=8 \pi\left({\tilde{\tau_{\alpha \beta}}}^{\cdot .} \frac{1}{2}\left(t_{\alpha \beta}+\lambda n_{\alpha} n_{\beta}\right) \tau_{\delta}^{\delta \bullet}\right) \text {. }
$$

A shell with isotropic internal pressure is characterized by the energy-momentum tensor

$$
\tau^{\alpha \beta}=(\sigma+\lambda p) u^{\alpha} u^{\beta}+p\left(s^{\alpha \beta}-n_{\bullet}^{\alpha} n_{\bullet}^{\beta}\right),
$$

where the fields $u^{\alpha}, \sigma, p$ are defined only on $\Sigma$ and $u^{\alpha}$ is tangential to $\Sigma$. Hence a fluid shell is characterized by a certain algebraic structure of the jump of the secondfundamental form at $\Sigma$.

In Einstein's theory the vacuum field equations on the complement of $\Sigma$ imply, at the shell,

$$
\tau_{\mid \beta}^{\alpha \beta}
$$

and

$$
\left({ }^{+} k_{\alpha \beta}+{ }^{-} k_{\alpha \beta}\right) \tau^{\alpha \beta}=0 .
$$

These equations are also meaningful for $\lambda=0$, but do not follow, and we postulate them in this case. Hence properties (1), (2), and the conditions (2.4), (2.5), (2.6), and (2.7) define a shell with isotropic internal pressure in the frame theory. In Appendix A it is shown that in this way we do obtain a Newtonian shell.

Let us consider as an example a static, spherically symmetric shell.

The time metric:

$$
\begin{aligned}
{ }^{+} t_{\alpha \beta} d x^{\alpha} d x^{\beta}= & \left(1-\frac{2 \lambda M^{ \pm}}{r}\right) d t^{2} \\
& -\lambda\left[\frac{d r^{2}}{1-\frac{2 \lambda M^{ \pm}}{r}}+r^{2}\left(d \theta^{2}+\sin ^{2} \theta d \phi^{2}\right)\right]
\end{aligned}
$$

(with $\lambda=c^{-2}, G=1$, we also write ${ }^{ \pm} t_{\alpha \beta}=-\lambda{ }^{ \pm} g_{\alpha \beta}$, which implies, for $\left.\lambda>0, s^{\alpha \beta}=g^{\alpha \beta}\right)$.

The space metric:

$$
\begin{gathered}
{ }^{ \pm} s^{\alpha \beta}:-\lambda\left(1-\frac{2 \lambda M^{ \pm}}{r}\right)^{-1}\left(\frac{\partial}{\partial t}\right)^{2}+\left(1-\frac{2 \lambda M^{ \pm}}{r}\right)\left(\frac{\partial}{\partial r}\right)^{2} \\
+r^{-2}\left[\left(\frac{\partial}{\partial \theta}\right)^{2}+\sin ^{-2}\left(\frac{\partial}{\partial \phi}\right)^{2}\right]
\end{gathered}
$$

We assume $2 M^{-}<2 M^{+}<R$, where $R$ is the area radius of the shell.

We use proper time $T$ at the shell as a time coordinate at both sides of the shell $s$ which makes the identification from both sides easy, $(T, \theta, \phi, r)=\left(x^{\alpha}\right),(T, \theta, \phi)=\left(x^{a}\right)$ :

$$
\begin{gathered}
g_{\alpha \beta}=-\lambda^{-1} e^{2 \Phi} d T^{2}+r^{2} d \Sigma^{2}+e^{2 \Lambda} d r^{2}, \\
e^{2 \Phi}=\frac{1-\frac{2 \lambda M}{r}}{1-\frac{2 \lambda M}{R}}, \quad e^{2 \Lambda}=\frac{1}{1-\frac{2 \lambda M}{r}} .
\end{gathered}
$$

$\Lambda^{+}(R) \neq \Lambda^{-}(R)$ if the masses are different at both sides. $T, \theta, \phi$ are coordinates on the shell. (In these coordinates the metric is not continuous at the shell. Constant rescaling of the metric on one side would give a continuous metric. This is, however, not needed.) We shall write 


$$
e^{-2 \Lambda}=F^{2}=1-\frac{2 \lambda M}{r}, \quad 2 F F^{\prime}=\frac{2 \lambda M}{r^{2}} .
$$

Second-fundamental form: we normalize $s^{\alpha \beta} n_{\alpha} n_{\beta}=1, n_{\alpha}$ $=\left(0,0,0, F^{-1}\right)$ :

$$
\begin{gathered}
k_{a b}=-\frac{1}{2}\left[F g_{a b, r}\right], \\
k_{00}=F^{-1} \frac{M}{R^{2}}, \\
k_{\theta \theta}=-F R, \\
k_{\phi \phi}=-F R \sin ^{2} \theta, \\
\Delta k_{00}={ }^{+} k_{00}-{ }^{-} k_{00}=\left(\frac{M^{+}}{F^{+}}-\frac{M^{-}}{F^{-}}\right) R^{-2}, \\
\Delta k_{\theta \theta}={ }^{+} k_{\theta \theta^{-}}{ }^{-} k_{\theta \theta}=-\left(F^{+}-F^{-}\right) R, \\
\Delta k_{\phi \phi}={ }^{+} k_{\phi \phi}-{ }^{-} k_{\phi \phi}=-\left(F^{+}-F^{-}\right) R \sin ^{2} \theta .
\end{gathered}
$$

The matter tensor of the shell is

$$
\begin{aligned}
& \tau^{\alpha \beta}=(\sigma+\lambda p) u^{\alpha} u^{\beta}+p\left(s^{\alpha \beta}-n_{.}^{\alpha} n_{\bullet}^{\beta}\right), \\
& \tau_{\alpha \beta}^{\ddot{*}}=\tau^{\kappa \sigma} t_{\kappa \alpha} t_{\beta \sigma}, \\
& n_{\alpha}=\left(0,0,0, F^{-1}\right), \quad n_{\bullet}^{\alpha}=s^{\alpha \beta} n_{\beta}=(0,0,0, F), \\
& u^{\alpha}=(1,0,0,0), \quad t_{\alpha \beta} u^{\alpha} u^{\beta}=1, \\
& \tau^{00}=\sigma, \quad \tau^{\theta \theta}=p R^{-2}, \quad \tau^{\phi \phi}=p R^{-2} \sin ^{-2} \theta, \\
& \tau_{00}=\sigma, \quad \ddot{\tau_{\theta \theta}}=\lambda^{2} p R^{2}, \quad \ddot{\tau_{\phi \phi}}=\lambda^{2} p R^{2} \sin ^{2} \theta, \\
& \tau_{0}^{0 \cdot}=\sigma, \quad \tau_{\theta}^{\theta_{\theta}^{*}}=-\lambda p, \quad \phi_{\phi}^{\phi{ }^{\bullet}}=-\lambda p, \\
& \tau_{\mu}^{\mu \cdot}=\sigma-2 \lambda p .
\end{aligned}
$$

The field equation at the shell is

$$
\Delta k_{\alpha \beta}=8 \pi\left(\tilde{\tau}_{\alpha \beta}^{\cdot}-\frac{1}{2}\left(t_{\alpha \beta}+\lambda n_{\alpha} n_{\beta}\right) t_{\mu}^{\mu}\right) .
$$

This gives

$$
\begin{gathered}
\Delta k_{00}=\left(\frac{M^{+}}{F^{+}}-\frac{M^{-}}{F^{-}}\right) R^{-2}=8 \pi\left(\frac{1}{2} \sigma+\lambda p\right), \\
\Delta k_{\theta \theta}=-\left(F^{+}-F^{-}\right) R=8 \pi \frac{1}{2} \lambda p R^{2} .
\end{gathered}
$$

The equilibrium condition of the shell is

$$
\left({ }^{+} k_{\alpha \beta}+{ }^{-} k_{\alpha \beta}\right) \tau^{\alpha \beta}=0,
$$

and hence

$$
\left(\frac{M^{+}}{F^{+}}-\frac{M^{-}}{F^{-}}\right) R^{-2} \sigma-2\left(F^{+}+F^{-}\right) R p R^{-2}=0 .
$$

The metrics (2.8) and (2.9) parametrized by $\lambda$ describe a one-parameter family with a Newtonian limit. For the metrics, this is obvious: for the connection, it can be checked easily. The equilibrium condition reduces for $\lambda=0$ to

$$
\left(M^{+}-M^{-}\right) R^{-2} \sigma-4 p R^{-1}=0,
$$

which is obviously the correct Newtonian result for the case $M^{-}=0$. In the general case $M^{+}$is the total mass (far field), and hence $M^{+}=M^{-}+M^{S}$, where $M^{S}$ is the mass of the shell. Again, we have the correct Newtonian relation. (The field equation expresses the various masses in terms of the densities.) Nonstatic, spherically symmetric shells are studied, for example, in [1], [7].

\section{LINEARIZATION AND THE SHELL TRANSITION CONDITIONS}

In this section I want to calculate the oscillation around a static shell to first order. The case of radial oscillations is treated in [6]. The background spacetime, a shell around a black hole or a body, is a Schwarzschild spacetime with mass $\mathrm{M}^{-}$inside the shell and with mass $\mathrm{M}^{+}$outside:

$$
\begin{aligned}
& \bar{g}_{00}=-\lambda^{-1}\left(1-\frac{2 \lambda M^{ \pm}}{r}\right)\left(1-\frac{2 \lambda M^{ \pm}}{R}\right)^{-1}=-\lambda^{-1} e^{2 \Phi^{ \pm}}, \\
& \bar{g}_{\theta \theta}=r^{2}, \\
& \bar{g}_{\phi \phi}=r^{2} \sin ^{2} \theta, \\
& \bar{g}_{r r}=\left(1-\frac{2 \lambda M^{ \pm}}{r}\right)^{-1}=e^{2 \Lambda^{ \pm}} .
\end{aligned}
$$

I take the same notation for the perturbations as in Kind's thesis [12]. The nonvanishing perturbation fields are (I consider only $\phi$-independent even perturbations)

$$
\begin{aligned}
& \delta g_{00}=-\lambda^{-1} e^{2 \Phi} 2 k Y=-e^{2 \Phi} 2 \hat{k} Y, \quad \lambda^{-1} k=\hat{k}, \\
& \delta g_{\theta \theta}=r^{2}(2 s Y+2 a Y, \theta \theta, \\
& \delta g_{\phi \phi}=r^{2}(2 s Y+2 a \cot \theta Y, \theta) \sin ^{2} \theta, \\
& \delta g_{r r}=e^{2 \Lambda} 2 \mu Y, \\
& \delta g_{0 r}=\lambda^{-1 / 2} e^{\Lambda+\Phi} \eta Y, \\
& \delta g_{0 \theta}=\lambda^{-1 / 2} e^{\Phi} r \xi Y_{, \theta}, \quad \lambda^{-1 / 2} \xi=\hat{\xi}, \\
& \delta g_{r \theta}=e^{\Lambda} r \omega Y_{, \theta} .
\end{aligned}
$$

The perturbations of the time metric are

$$
\delta t_{\alpha \beta}=-\lambda \delta g_{\alpha \beta} .
$$

We use a Gaussian gauge to calculate the perturbations of the second-fundamental forms on both sides of the shell. Imag- 
ine a one-parameter family of shells. Then we can introduce on each member a Gaussian coordinate system relative to the shell. Linearization of such a family implies

$$
\eta=\omega=\mu=0
$$

On the shell we have the fluid flow vector $u^{\alpha}$. Because of the $Y_{l m}$ behavior we choose, this flow is irrotational, and hence we can use the flow lines and some coordinates in the orthogonal two-surface as coordinates. Hence

$$
\xi=0 \text { for } r=R \text {. }
$$

On the shell, $k, s, a$ are continuous. Their radial derivatives, however, will have just limits from both sides satisfying certain jump conditions, which we will calculate now.

For the perturbations of the second-fundamental form, we obtain

$$
\begin{aligned}
\delta k_{a b} & =-\frac{1}{2} F \delta g_{a b, r}, \\
\delta k_{00} & =+\frac{1}{2} F\left[e^{2 \Phi} 2 \hat{k}\right]_{, r} Y, \\
\delta k_{\theta \theta} & =-\frac{1}{2} F\left[r^{2}\left(2 s Y+2 a Y_{, \theta \theta}\right)\right]_{, r}, \\
\delta k_{\phi \phi} & =-\frac{1}{2} F\left[r^{2}\left(2 s Y+2 a \cot \theta Y_{, \theta}\right) \sin ^{2} \theta\right]_{, r}, \\
\delta k_{0 \theta} & =-\frac{1}{2} F\left[e^{\Phi} r \hat{\xi}\right]_{, r} Y,_{, \theta} .
\end{aligned}
$$

The perturbation of the energy-momentum tensor of the shell is

$$
\begin{aligned}
& \delta\left[\tau^{\alpha \beta}\right]=\delta\left[(\sigma+\lambda p) u^{\alpha} u^{\beta}+p\left(s^{\alpha \beta}-n_{\bullet}^{\alpha} n_{\bullet}^{\beta}\right)\right] \\
& \delta \tau^{\alpha \beta=} \delta(\sigma+\lambda p) \bar{u}^{\alpha} \bar{u}^{\beta}+(\bar{\sigma}+\lambda \bar{p}) \delta\left(u^{\alpha} u^{\beta}\right)+(\delta p) \bar{s}^{\alpha \beta} \\
&+\bar{p} \delta s^{\alpha \beta}-(\delta p) \bar{n}_{\bullet}^{\alpha} \bar{n}_{\bullet}^{\beta}-\bar{p} \delta\left(n_{\bullet}^{\alpha} n_{\bullet}^{\beta}\right) .
\end{aligned}
$$

The four-velocity is normalized, $t_{\alpha \beta} u^{\alpha} u^{\beta}=1$. As $\bar{u}^{\alpha}=\delta_{0}^{\alpha}$, $\bar{t}_{00}=1$, we obtain $\delta t_{00}+2 \delta u^{0}=0$ or

$$
\delta u^{0}=-\frac{1}{2} \delta t_{00}=\lambda e^{2 \Phi} \hat{k} Y .
$$

$\delta\left(u^{\alpha} u^{\beta}\right)$ has only a 00-component $\delta\left(u^{0} u^{0}\right)=-\delta t_{00}$. We need also

$$
\delta s^{\alpha \beta}=\delta g^{\alpha \beta}=-g^{\alpha \mu} g^{\beta \nu} \delta g_{\mu \nu} .
$$

Since we use a Gaussian foliation for the one-parameter family defining the linearization, we have $\delta n_{\alpha}=0, \delta s^{r r}=0$, which implies $\delta n_{\bullet}^{\alpha}=0$. Hence we obtain $($ at $r=R)[\delta[\sigma]$ $=\delta \sigma Y(\theta)]$

$$
\begin{aligned}
\delta \tau^{00}= & (\delta \sigma+\lambda \delta p) Y+(\bar{\sigma}+\lambda \bar{p})\left(-\delta t_{00}\right)+\delta p(-\lambda) \\
& +\bar{p} \lambda^{2} \delta g_{00} \\
\delta \tau^{00}= & \delta \sigma Y+(\bar{\sigma}+\lambda \bar{p})\left(-\delta t_{00}\right)-\bar{p} \lambda \delta t_{\infty} \\
= & \delta \sigma Y-\delta t_{00}(\bar{\sigma}+2 \lambda \bar{p}) \\
\delta \tau^{\theta \theta}= & \delta p Y \bar{s}^{\theta \theta}+\bar{p} \delta s^{\theta \theta} \\
\delta \tau^{\theta \theta}= & \delta p Y R^{-2}-\bar{p} R^{-4} \delta g_{\theta \theta}, \\
\delta \tau^{\phi \phi}= & \delta p Y R^{-2} \sin ^{-2} \theta-\bar{p} R^{-4} \delta g_{\phi \phi} \sin ^{-4} \theta
\end{aligned}
$$

For the linearized shell we need, in the transition conditions (2.4),

$$
\begin{aligned}
A_{\alpha \beta}:= & \delta\left(\tau_{\alpha \beta}^{\ddot{\theta}}-\frac{1}{2}\left(t_{\alpha \beta}-\lambda n_{\alpha} n_{\beta}\right) t_{\mu}^{\mu \cdot}\right), \\
A_{\alpha \beta}= & \delta \tau_{\alpha \beta}^{\ddot{*}}-\frac{1}{2}\left[\delta t_{\alpha \beta}-\lambda \delta\left(n_{\alpha} n_{\beta}\right)\right] \bar{t}_{\mu}^{\mu \bullet} \\
& -\frac{1}{2}\left(\bar{t}_{\alpha \beta}-\lambda \bar{n}_{\alpha} \bar{n}_{\beta}\right) \delta t_{\mu}^{\mu \cdot} .
\end{aligned}
$$

We calculate first

$$
\begin{aligned}
\delta\left({\tilde{\tau_{\alpha \beta}}}_{\alpha \beta}\right) & =\delta\left(\tau^{\mu \nu} t_{\alpha \mu} t_{\beta \nu}\right) \\
& =\delta \tau^{\mu \nu} \bar{t}_{\alpha \mu} \bar{t}_{\beta \nu}+\bar{\tau}^{\mu \nu}\left(\delta t_{\alpha \mu} \bar{t}_{\beta \nu}+\bar{t}_{\alpha \mu} \delta t_{\beta \nu}\right) .
\end{aligned}
$$

For $a=0, \theta, \phi$ we obtain

$$
\delta\left(\tau_{a a}^{\ddot{*}}\right)=\delta t^{a a} \bar{t}_{a a} \bar{t}_{a a}+\bar{\tau}^{a a} 2 \bar{t}_{a a} \delta t_{a a} .
$$

Linearization of the trace gives

$$
\delta\left(\tau_{\alpha}^{\alpha \bullet}\right)=\delta\left(t^{\alpha \beta} t_{\alpha \beta}\right)=\delta(\sigma-2 \lambda p)=(\delta \sigma-2 \lambda \delta p) Y
$$

For $a=0, \theta, \phi$ we have

$$
\begin{aligned}
A_{a a}= & \left(\delta \tau^{a a} \bar{t}_{a a} \bar{t}_{a a}+2 \delta t_{a a} \bar{\tau}^{a a} \bar{t}_{a a}-\frac{1}{2} \delta t_{a a}(\bar{\sigma}-2 \lambda \bar{p})\right. \\
& \left.-\frac{1}{2} \bar{t}_{a a}(\delta \sigma-2 \lambda \delta p) Y\right)
\end{aligned}
$$

and hence 


$$
\begin{aligned}
& A_{00}=\frac{1}{2} \delta \sigma Y+\lambda \delta p Y+\delta t_{00}\left(\frac{1}{2} \bar{\sigma}-\lambda \bar{p}\right), \\
& A_{\theta \theta}=8 \pi\left(2 \lambda^{2} R^{2} \delta p Y+\delta g_{\theta \theta} \frac{1}{2} \lambda \bar{\sigma}\right), \\
& A_{\phi \phi}=8 \pi\left(2 \lambda^{2} R^{2} \delta p Y+\delta g_{\phi \phi} \frac{1}{2} \lambda \bar{\sigma}\right) .
\end{aligned}
$$

Furthermore,

$$
A_{0 \theta}=0 .
$$

Now we can finally write down the transition conditions (2.4), $\Delta k_{\alpha \beta}=8 \pi A_{\alpha \beta}$, which define a linearized fluid shell in the Gaussian comoving gauge:

$$
\begin{aligned}
\Delta \delta k_{00} & =+\Delta \frac{1}{2} F\left[{ }^{2 \Phi} 2 \hat{k}\right]_{, r} Y \\
& =8 \pi\left[\frac{1}{2} \delta \sigma Y+\lambda \delta p Y-\lambda \delta g_{00}\left(\frac{1}{2} \bar{\sigma}-\lambda \bar{p}\right)\right], \\
\Delta \delta k_{\theta \theta} & =-\Delta \frac{1}{2} F\left[r^{2}\left(2 s Y+2 a Y_{, \theta \theta}\right)\right]_{, r} \\
& =8 \pi\left(2 \lambda^{2} R^{2} \delta p Y+\delta g_{\theta \theta} \frac{1}{2} \lambda \bar{\sigma}\right), \\
\Delta \delta k_{\phi \phi} & =-\Delta \frac{1}{2} F\left[r^{2}\left(2 s Y+2 a \cot \theta Y{ }_{, \theta}\right) \sin ^{2} \theta\right]_{, r} \\
& =8 \pi\left(2 \lambda^{2} R^{2} \delta p Y+\delta g_{\phi \phi} \frac{1}{2} \lambda \bar{\sigma}\right), \\
\Delta \delta k_{0 \theta} & =-\Delta \frac{1}{2} F \lambda^{-1 / 2}\left[e^{\Phi} r \xi\right]_{, r} Y_{, \theta}=0 .
\end{aligned}
$$

Because of the linear independence of $Y$ and $Y_{\theta \theta}$, it holds that

$$
\begin{gathered}
\Delta F\left[e^{2 \Phi} \hat{k}_{G}\right]_{, r}+8 \pi \lambda\left(\frac{1}{2} \bar{\sigma}-\lambda \bar{p}\right) e^{2 \Phi} 2 \hat{k}_{G} \\
=8 \pi\left(\frac{1}{2} \delta \sigma+\lambda \delta p\right), \\
-\Delta F\left[r^{2} s_{G}\right]_{, r}-8 \pi \lambda \bar{\sigma} R^{2} s_{G}=8 \pi 2 \lambda^{2} R^{2} \delta p, \\
-\Delta F\left[r^{2} a\right]_{, r}-8 \pi \lambda \bar{\sigma} R^{2} a=0, \\
-\Delta F\left[e^{\Phi} r \hat{\xi}\right]_{, r}=0 .
\end{gathered}
$$

We have shown the following.

Suppose $a^{ \pm}, s^{ \pm}, \hat{k}^{ \pm}$, and $\bar{\xi}^{ \pm}$satisfy the linearized vacuum field equations together with $\eta=\omega=\mu=0$ (Gaussian gauge), $a^{ \pm}, s^{ \pm}, \hat{k}^{ \pm}$, and $\bar{\xi}^{ \pm}$are continuous at $R$, the background shell, and $a^{\prime \pm}, s^{\prime \pm}, \bar{k}^{\prime \pm}$, and $\bar{\xi}^{\prime \pm}$, the radial derivatives at both sides of the shell, satisfy Eqs. (3.21)(3.24) for some $\delta \sigma, \delta p$. Then these fields represent a linearly oscillating shell. If an equation of state, $\delta p=\alpha \delta \sigma$, is prescribed for the oscillation, one can eliminate these matter fields from Eqs. (3.21), (3.22), and three transition conditions remain.

Later, in the existence proof, we will need the transition condition in Regge-Wheeler gauge which we shall calculate next. From Appendix $\mathrm{C}$ we find the following expressions of Regge-Wheeler perturbations in terms of the Gauss perturbations used up to now:

$$
\begin{gathered}
-\mu=k=k_{G}-\frac{1}{c^{2}} e^{-2 \Phi} r^{2} \ddot{a}_{G}+\frac{1}{c} e^{-\Phi} r \xi_{G}+e^{-2 \Lambda} r^{2} \Phi^{\prime} a_{G}^{\prime}, \\
\mu=e^{-2 \Lambda} r^{2}\left[a_{G}^{\prime \prime}+a_{G}^{\prime}\left(\frac{2}{r}-\Lambda^{\prime}\right)\right] \\
s=s_{G}+e^{-2 \Lambda} r a_{G}^{\prime} .
\end{gathered}
$$

From this we find, because $s_{G}$ and $a_{G}$ are continuous jumps for $s$ and $\mu$,

$$
\begin{gathered}
\Delta s:=s^{+}-s^{-}=e^{-2 \Lambda^{+}} r{a_{G}^{\prime+}-e^{-2 \Lambda^{-}} r a_{G}^{\prime-},}^{-{ }^{-}}, \\
-\Delta \mu:=e^{-2 \Lambda^{+}} r^{2} \Phi^{\prime+} a_{G}^{\prime+}-e^{-2 \Lambda^{-}} r^{2} \Phi^{\prime-} a_{G}^{\prime-} .
\end{gathered}
$$

If $\Phi^{+} \neq \Phi^{-}$, i.e., if there is really a background shell, we can solve for $a_{G}^{\prime+}$ and $a_{G}^{\prime-}$ in terms of $\Delta s$ and $\Delta \mu$ :

$$
a_{G}^{\prime+}=L^{+}(\Delta s, \Delta \mu), \quad a_{G}^{\prime-}=L^{-}(\Delta s, \Delta \mu) .
$$

The transition condition (3.23) for $a^{\prime}$ expresses $a$ linearly in terms of $a^{\prime+}, a^{\prime-}$; hence, we obtain

$$
a_{G}=L(\Delta s, \Delta \mu) .
$$

These relations can be differentiated in time.

Now we can express all the Gaussian quantities in terms of the Regge Wheeler quantities:

$$
\begin{aligned}
k_{G}= & -\mu^{+}+\frac{1}{c^{2}} e^{-2 \Phi^{+}} r^{2} \ddot{a}_{G}^{+}-\frac{1}{c} e^{-\Phi^{+}} r \dot{\xi}_{G}^{+} \\
& -e^{-2 \Lambda^{+}} r^{2} \Phi^{\prime+} a_{G}^{\prime+}
\end{aligned}
$$

(we could also use the - side to determine $k_{G}$ ). Differentiation gives $k_{G}^{\prime}$ in terms of Regge-Wheeler quantities:

$$
\begin{aligned}
k_{G}^{\prime+}= & -\mu^{\prime+}+\frac{1}{c^{2}} e^{-2 \Phi^{+}} r^{2} \ddot{a}_{G}^{\prime+}+\left(\frac{1}{c^{2}} e^{-2 \Phi^{+}} r^{2}\right)^{\prime} \ddot{a}_{G}^{+} \\
& -\frac{1}{c} e^{-\Phi^{+}} r \dot{\xi}_{G}^{\prime+}-\frac{1}{c}\left(e^{-\Phi^{+}} r\right)^{\prime} \dot{\xi}_{G}^{+}-e^{-2 \Lambda^{+}} r^{2} \Phi^{\prime+} a_{G}^{\prime \prime} \\
& -\left(e^{-2 \Lambda^{+}} r^{2} \Phi^{\prime+}\right)^{\prime} a_{G}^{\prime+} .
\end{aligned}
$$


In the transition condition for $k^{\prime}$, the $\xi^{\prime}$ terms drop out because of the $\xi$ transition condition (3.24). We need $a_{G}^{\prime \prime+}$ in terms of Regge-Wheeler quantities. We use the second $\mu$ equation (3.26) to calculate $a_{G}^{\prime \prime}$ :

$$
a_{G}^{\prime \prime+}=r^{-2} e^{2 \Lambda^{+}} \mu^{+}-\left(\frac{2}{r}-\Lambda^{+}\right) a_{G}^{\prime+}
$$

or

$$
a_{G}^{\prime \prime+}=r^{-2} e^{2 \Lambda^{+}} \mu^{+}-\left(\frac{2}{r}-\Lambda^{+}\right) L^{+}(\Delta s, \Delta \mu) .
$$

Similarly, for $s$,

$$
\begin{gathered}
s_{G}=s^{+}-e^{-2 \Lambda^{+}} r a_{G}^{\prime+}, \\
s_{G}^{\prime+}=s^{\prime+}-e^{-2 \Lambda^{+}} r{a_{G}^{\prime \prime}}^{+}-\left(e^{-2 \Lambda^{+}} r\right)^{\prime} a_{G}^{\prime+} .
\end{gathered}
$$

Now we can rewrite the transition conditions (3.21), (3.22) in Regge-Wheeler quantities $s$ and $\mu=k$. We obtain one condition if an equation of state $\delta p=\alpha \delta \sigma$ is given.

To express the $\xi$ transition condition (3.24), $-\Delta \frac{1}{2} F \lambda^{-1 / 2}\left[e^{\Phi} r \xi\right]_{, r}=0$, in $s$ and $\mu$, we use the $\delta R_{r \theta}$ field equation

$$
\begin{aligned}
0= & \delta R_{r \theta}=a_{G}^{\prime}-k_{G}^{\prime}-s_{G}^{\prime}-k_{G}\left(\Phi^{\prime}-\frac{1}{r}\right) \\
& +\frac{r}{2 c} e^{-\Phi}\left[-\dot{\xi}^{\prime}+\dot{\xi}\left(\frac{1}{r}-\Phi^{\prime}\right)\right] .
\end{aligned}
$$

Remembering $\xi(R)=0$ and $\Phi(R)=1$, we can express $\dot{\xi}_{, r}$ as

$$
\lambda^{-1 / 2} R \dot{\xi}^{\prime}=a_{G}^{\prime}-k_{G}^{\prime}-s_{G}^{\prime}-k_{G}\left(\Phi^{\prime}-\frac{1}{r}\right) .
$$

Therefore the time derivative of Eq. (3.24) implies

$$
\Delta F\left(a_{G}^{\prime}-k_{G}^{\prime}-s_{G}^{\prime}-k_{G}\left(\Phi^{\prime}-\frac{1}{r}\right)=0 .\right.
$$

Since we have only used the time derivative of the condition (3.24), we have to impose $-\Delta \frac{1}{2} F \lambda^{-1 / 2}\left[e^{\Phi} r \xi\right]_{, r}=0$ at some time $t_{0}$, which can be achieved by the remaining gauge freedom.

If we insert the Gaussian expressions in terms of the Regge-Wheeler quantities given above, we obtain a second condition in Regge-Wheeler quantities and we have shown the following.

The shell transition conditions in the Regge-Wheeler gauge are two linear relations for $s^{ \pm}, s^{\prime \pm}, k^{ \pm}$, and $k^{\prime \pm}$ if an equation of state is given.

We do not write out these lengthy expressions here because the explicit expressions are not needed.

\section{EXISTENCE}

In this section we want to show how the existence of linearized solutions satisfying the linearized shell transition conditions can be established using the method of characteristics as in [8]. The convergence proof given in that paper can easily be adapted to the case treated here. The essential step is to check that the transition conditions give the information needed to perform the iteration and to control convergence.

We rewrite for convenience the system given in [8] (with $f=\mu, g=s$ ), which is equivalent to the field equations in Regge-Wheeler gauge. There are two wave equations for $s$ and $\mu$ :

$$
\begin{aligned}
& -c^{-2} e^{2(\Lambda-\Phi)} \ddot{s}+s^{\prime \prime}+s^{\prime}\left(\Phi^{\prime}-\Lambda^{\prime}+\frac{2}{r}\right)-e^{2 \Lambda} \frac{\lambda}{r^{2}} s \\
& +s \frac{2}{r}\left(\Phi^{\prime}-\Lambda^{\prime}+\frac{1}{r}\right)+\mu \frac{2}{r}\left(\Phi^{\prime}+\Lambda^{\prime}-\frac{1}{r}\right)=0 \\
& -c^{-2} e^{2(\Lambda-\Phi)} \ddot{\mu}+\mu^{\prime \prime}+\mu^{\prime}\left(5 \Phi^{\prime}-\Lambda^{\prime}-\frac{2}{r}\right)-e^{2 \Lambda} \frac{\lambda}{r^{2}} \mu \\
& -4 s^{\prime}\left(\Phi^{\prime}-\frac{1}{r}\right)+4 \mu\left(\Phi^{\prime \prime}+2 \Phi^{\prime} \Phi^{\prime}-\Phi^{\prime} \Lambda^{\prime}\right)=0
\end{aligned}
$$

and a constraint

$$
\begin{aligned}
Z:= & s^{\prime \prime}-s^{\prime}\left(\Lambda^{\prime}-\frac{3}{r}\right)-\frac{1}{r} \mu^{\prime}-e^{2 \Lambda} \frac{\lambda}{2 r^{2}}(s+\mu) \\
& +(s-\mu) \frac{1}{r}\left(\Phi^{\prime}-\Lambda^{\prime}+\frac{1}{r}\right)=0 .
\end{aligned}
$$

There is an ordinary differential equation for $\eta$,

$$
(2 c)^{-1} e^{(\Lambda-\Phi)} \dot{\eta}+s^{\prime}-\mu^{\prime}-2 \Phi^{\prime} \mu=0,
$$

and two more field equations

$$
\begin{aligned}
0= & Z_{1}:=c^{-1} e^{(\Lambda-\Phi)}\left[\dot{s}^{\prime}-\dot{s}\left(\Phi^{\prime}-\frac{1}{r}\right)-\frac{1}{r} \dot{\mu}\right] \\
& -e^{2 \Lambda} \frac{l(l+1)}{4 r^{2}} \eta, \\
0= & Z_{2}:=\frac{1}{2} c^{-1} e^{(\Lambda-\Phi)}(\dot{\mu}+\dot{s})-\frac{1}{4}\left(\eta^{\prime}+2 \Phi^{\prime} \eta\right) .
\end{aligned}
$$

Sufficiently differentiable solutions of Eqs. (4.1), (4.2) satisfy

$$
\begin{gathered}
-c^{-2} e^{2(\Lambda-\Phi)} \ddot{Z}+Z^{\prime \prime}+Z^{\prime}\left(5\left(\Phi^{\prime}-\Lambda^{\prime}\right)+\frac{2}{r}\right)-e^{2 \Lambda} \frac{\lambda}{r^{2}} Z \\
+2 Z\left(\Phi^{\prime \prime}-\Lambda^{\prime \prime}+3\left(\Phi^{\prime}-\Lambda^{\prime}\right)^{2}+\frac{4}{r} \Phi\right)=0
\end{gathered}
$$


and

$$
\begin{gathered}
c^{-1} e^{(\Lambda-\Phi)} \dot{Z}_{1}=Z^{\prime}+4 \Phi^{\prime} Z, \\
c^{-1} e^{(\Lambda-\Phi)} \dot{Z}_{2}=Z .
\end{gathered}
$$

The existence of solutions away from the shell is obvious: Cauchy data $s, \dot{s}, \mu, \dot{\mu}$ determine a solution of the two wave equations. If $Z=Z_{1}=Z_{2}=0$ holds for the data, it will also hold for the evolution of the data. The problem is to find solutions near the shell at $R$ which satisfy the transition conditions.

As in the case of stellar oscillation [8], existence will be demonstrated by showing that the transition conditions give the information needed to determine the relevant information along the characteristics outgoing at the boundary from the incoming quantities, as explained in [8].

As in [8], we will use $Z=0$ as a boundary condition on both sides. This will guarantee that a solution $s, \mu$ of the wave equations such that the data satisfy $Z=0$ satisfies identically $Z=0$ because $Z$ satisfies the homogeneous wave equation (4.7).

The equation

$$
\begin{gathered}
c^{-2} e^{(\Lambda-\Phi)} \ddot{s}-s^{\prime}\left(\Phi^{\prime}-\frac{1}{r}\right)-\frac{1}{r} \mu^{\prime}+e^{2 \Lambda} \frac{l(l+1)}{2 r^{2}}(s-\mu) \\
-s\left(\frac{2}{r} \Phi^{\prime}+\frac{1}{r^{2}}\right)-\mu\left(\frac{2}{r} \Phi^{\prime}-\frac{1}{r^{2}}\right)=0
\end{gathered}
$$

is a consequence of Eqs. (4.1), (4.2), and (4.3). It implies $Z$ $=0$ at the boundary if the wave equations hold and the data satisfy $Z=0$. To use $\ddot{s}$ as boundary data we have to differentiate the wave equation for $s$ with respect to the time variable.

At the left side of the shell, the relation

$$
{ }^{-} \ddot{s}-{ }^{-} \dot{s}^{\prime}=-\left({ }^{-} \ddot{s}+{ }^{-} \dot{s}^{\prime}\right)+2^{-} \ddot{s}
$$

shows how the field in the direction of the left-going characteristic is determined by the value in the direction of the right-going characteristic and the boundary values $\ddot{s}$, similarly for the outside.

For $\mu$ we shall show that the two transition conditions in Regge-Wheeler gauge are equivalent to the relations

$$
\begin{aligned}
+\ddot{\mu} & ={ }^{-} \ddot{\mu}+J \text { (lower derivatives), } \\
{ }^{+} F^{+} \dot{\mu}^{\prime} & ={ }^{-} F^{-} \dot{\mu}^{\prime}+J \text { (lower derivatives), }
\end{aligned}
$$

which imply

$$
{ }^{+} \ddot{\mu} \pm{ }^{+} F^{+} \dot{\mu}^{\prime}={ }^{-} \ddot{\mu} \pm^{-} F^{-} \dot{\mu}^{\prime}+J \text { (lower derivatives). }
$$

With these boundary conditions existence can be shown as in [8].

It remains to establish the desired form of the conditions for $\mu$.
We can write the the transition conditions (3.21), (3.22) in the Gauss gauge in the form

$$
{ }^{+} F^{+} \hat{k}_{G}^{\prime}-{ }^{-} F^{-} k_{G}^{\prime}+A_{1} \hat{k}_{G}=A_{2}\left(\alpha, s_{G}{ }^{ \pm \prime}, s_{G}{ }^{ \pm}, \lambda, R\right) .
$$

The transition condition for $\xi$ in the form (3.40) is

$$
-{ }^{+} F^{+} \hat{k}_{G}^{\prime}-{ }^{-} F^{-} \hat{k}_{G}^{\prime}+A_{3} \hat{k}_{G}=A_{4}\left(s_{G}^{\prime}{ }^{ \pm}, a^{\prime \pm}\right) .
$$

Because of $A_{1} \neq A_{2}$, we can solve

$$
\begin{gathered}
{ }^{+} F^{+} \hat{k}_{G}^{\prime}-{ }^{-} F^{-} \hat{k}_{G}^{\prime}=A_{5}\left(C, s_{G}^{\prime}{ }^{ \pm}, s_{G}{ }^{ \pm}, \lambda, R, a^{\prime \pm}\right), \\
\hat{k}_{G}=A_{6}\left(C, s_{G}^{\prime}{ }^{ \pm}, s_{G}{ }^{ \pm}, \lambda, R, a^{\prime \pm}\right) .
\end{gathered}
$$

Now we replace the Gaussian quantities by Regge-Wheeler quantities according to Sec. III. In the terms with $\ddot{a}$ and $\ddot{a}^{\prime}$, we replace $s^{+}$and $s^{-}$by Eq. (4.9) and remarkably the wanted combination of $\mu^{\prime}$ appears:

$$
s^{+}-s^{-}=\lambda \frac{1}{R}\left({ }^{+} F^{+} \mu^{\prime}-{ }^{-} F^{-} \mu^{\prime}\right)+\text { lower order. }
$$

It can be checked that it is possible to solve in the two transition conditions for ${ }^{+} F^{+} \mu^{\prime}-{ }^{-} F^{-} \mu^{\prime}$ and $\ddot{\mu}^{-}-\ddot{\mu}^{-}$.

If we perform the time derivative to obtain $\ddot{\mu}^{ \pm}=$ ${ }^{ \pm} F^{ \pm} \dot{\mu}^{\prime}+\cdots$, second-order terms ${ }^{ \pm} \dot{s}^{\prime}$ appear. We eliminate these by the field equation $Z_{1}=0$, which introduces $\eta$ into the boundary conditions. Therefore we have to take Eq. (4.4) for $\eta$ along with the two wave equations in the iteration to prove the existence as in [8].

Let me finally outline how one can construct data of the following two types.

First, we want a gravitational wave which falls onto the shell. To find such a solution of our constraints, it is simplest to use Moncrief's gauge-invariant and constraint-free formulation of perturbations of Schwarzschild. In this approach one chooses $\Psi$ and $\dot{\Psi}$ unconstraints and these data determine the solutions $\Psi$ of a wave equation. From $\Psi$ one calculates by differentiation and algebraic manipulation the fields in the Regge-Wheeler gauge. Hence we can take the data for $\Psi$ such that they vanish outside an interval not containing the shell. The corresponding solution describes a situation in which gravitational waves impinge from the inside or outside onto the shell and will set it into motion.

Second, we would like to have the opposite situation, an initial $\delta \sigma$ and as little gravitational waves as possible. Take data for $\Psi$ which vanishes outside an interval around the shell. We have just to make sure that we choose the values and derivatives of $\Psi$ at both sides in such a way that $\delta \sigma$ does not vanish. This is possible because we have just two linear transition conditions in the Regge-Wheeler gauge (see the end of Sec. III). 


\section{QUASINORMAL MODES}

To find the quasinormal modes of the shell, one has to proceed as follows [10]. First, one determines solutions with $e^{s t}$ time behavior for any complex $s$. Local solutions of the two wave equations (4.1), (4.2) are determined by initial values $s, s^{\prime}, \mu, \mu^{\prime}$ at some point $r_{0}$. The condition that $Z$ and $Z^{\prime}$ vanish at this point gives two relations between those data. If they are satisfied, $Z=0$ holds for the solution and all field equations hold because of Eq. (4.8). Hence we have a twoparameter family of solutions on both sides of the shell. Regularity at the center (or the horizon) determines a oneparameter family. If we fix the overall constant at the center, we have just one solution for a given $s$. The two transition conditions at the shell determine uniquely the outer solution. Hence there is a unique solution for each $s$ with $e^{s t}$ time behavior.

If we use data for $\mu, \mu^{\prime}$ to characterize the local solutions, we can take the transition conditions (4.11), (4.12) for $\mu, \mu^{\prime}$ from Sec. IV.

Similarly, one can find for real $s$ a unique solution which satisfies the correct boundary condition at infinity. From two solutions-one regular at the center, one with the correct behavior at infinity for each real $s$-one builds the Green function first for real $s$. The analytic extension of the Green function has poles, the quasinormal modes.

In numerical calculations of quasinormal modes, one starts with the solution regular at the center for complex $s$ and tries to find those values of $s$ for which the solution satisfies at infinity the "outgoing wave condition": the solution for general complex $s$ is near infinity, a linear combination of two solutions with the property that for real $s$ one solution is bounded the other is diverging. "Outgoing", is defined by the property that only the part bounded for real $s$ is present.

The numerical calculations of the quasinormal modes are left to a further paper.

\section{NEWTONIAN LIMIT OF THE EQUATIONS}

A solution of the linearized vacuum field equations which satisfies the shell transition condition describes a nonradially oscillating shell. In this section, I want to consider the Newtonian limit of the field equations and the transition condition, and it will turn out that the limiting system really is a formulation of the Newtonian problem. There is a conceptual difference between shells in Newton's and Einstein's theory: In Einstein's theory the "main action" is in the field equations outside the shell and the shell quantities appear via the transition conditions. In Newton's theory the field outside the shell is just the Newtonian gravitational field and the equations of motion are formulated at the shell. Hence to do successfully the Newtonian limit of the equations we have to extract from the field equations the relevant relations (2.5) and (2.6) at the shell.

The object available in both theories is the connections. If we look at the linearized connection components in Appen$\operatorname{dix} \mathrm{B}$, one realizes that we only have a limit - in the Gaussian gauge-if we assume that $\lambda^{-1} k=\hat{k}$ and $\lambda^{-1 / 2} \xi$ are finite.
First, we calculate the linearization of the equations $\tau^{a b}{ }_{\| b}$ of the shell (we write $\|$ for the covariant derivative on the background shell):

$$
\tau^{\alpha \beta}=(\sigma+\lambda p) u^{\alpha} u^{\beta}+p\left(s^{\alpha \beta}-n_{\bullet}^{\alpha} n_{\bullet}^{\beta}\right),
$$

which implies, for $a=0, \theta, \phi$,

$$
\tau^{a b}=(\sigma+\lambda p) u^{a} u^{b}+p s^{a b} .
$$

$\tau^{a b}{ }_{\| b}$ splits as usual into the two equations

$$
\sigma_{, a} u^{a}+(\sigma+\lambda p) u^{b}{ }_{\| b}=0
$$

and

$$
(\sigma+\lambda p) u_{\| b}^{a} u^{b}+p_{, b} s^{a b}=0 .
$$

Linearization gives

$$
\begin{gathered}
\delta \sigma_{, a} \bar{u}^{a}+\bar{\sigma}_{, a} \delta u^{a}+(\delta \sigma+\lambda \delta p) \bar{u}_{\| b}^{b}+(\bar{\sigma}+\lambda \bar{p}) \delta u_{\| b}^{b}=0 \\
(\delta \sigma+\lambda \delta p) \bar{u}^{a}{ }_{\| b} \bar{u}^{b}+(\bar{\sigma}+\lambda \bar{p}) \delta\left(u_{\| b}^{a} \bar{u}^{b}\right) \\
+\delta p_{, b} \bar{s}^{a b}+\bar{p}_{, b} \delta s^{a b}=0
\end{gathered}
$$

If we use the background relations $0=\bar{\sigma}_{, a} \bar{u}^{a}=\bar{u}_{\| b}^{b}=\bar{p}_{, a}$ $=\bar{u}^{a}{ }_{\| b} \bar{u}^{b}$, we obtain

$$
\begin{gathered}
\delta \sigma_{, a} \bar{u}^{a}+(\bar{\sigma}+\lambda \bar{p}) \delta u_{\| b}^{b}=0, \\
(\bar{\sigma}+\lambda \bar{p}) \delta\left(u_{\| b}^{a} u^{b}\right)+\delta p_{, b} \bar{s}^{a b}=0 .
\end{gathered}
$$

With the linearized connection from Appendix B, we find

$$
\delta u^{a}{ }_{\| a}=[2 \dot{s}-l(l+1) \dot{a}] Y .
$$

The perturbed acceleration $\delta\left(u^{a}{ }_{\| b} u^{b}\right)$ has only a nonvanishing $\theta$ component

$$
\lambda^{-1} R^{-2} e^{2 \Phi} k Y_{, \theta}
$$

So we obtain finally, for the motion tangentially to the shell $(\delta \sigma=\widetilde{\sigma} Y)$,

$$
\begin{gathered}
\delta \dot{\tilde{\sigma}}+(\bar{\sigma}+\lambda \bar{p})[2 \dot{s}-l(l+1) \dot{a}]=0, \\
(\bar{\sigma}+\lambda \bar{p}) \lambda^{-1} R^{-2} e^{2 \Phi} k Y_{, \theta}+\delta p_{, \theta} R^{-2}=0 .
\end{gathered}
$$

The "normal motion" of the shell is contained in the equation

$$
\delta\left[\left({ }^{+} k_{\alpha \beta}+{ }^{-} k_{\alpha \beta}\right) \tau^{\alpha \beta}\right]=0
$$

With Eqs. (3.6), (3.11) we obtain 


$$
\begin{gathered}
{\left[\left(\frac{1}{2} F\left[e^{2 \Phi} 2 \hat{k}\right]_{, r} Y\right)^{+}+\left(\frac{1}{2} F\left[e^{2 \Phi} 2 \hat{k}\right]_{, r} Y\right)^{-}\right] \bar{\sigma}} \\
+\left(\frac{M^{+}}{\sqrt{F^{+}}}+\frac{M^{-}}{\sqrt{F^{-}}}\right) R^{-2}\left[\delta \sigma-\delta t_{00}(\bar{\sigma}+2 \lambda \bar{p})\right] \\
+\left[\left(-\frac{1}{2} F r^{2}[4 s-2 l(l+1) a]\right)_{, r}^{+}\right. \\
\left.+\left(-\frac{1}{2} F r^{2}[4 s-2 l(l+1) a]\right)_{, r}^{-}\right] \bar{p} R^{-2} Y \\
\quad-4 \delta p R^{-1}+4 \bar{p} R^{-1}[2 s-l(l+1) a] Y=0 .
\end{gathered}
$$

Now we can evaluate Eqs. (6.11), (6.12), and (6.14) for $\lambda$ $=0$ to obtain the Newtonian equations. Remembering $\lambda^{-1} k=\hat{k}$, we get

$$
\begin{gathered}
\delta \dot{\tilde{\sigma}}+\bar{\sigma}[2 \dot{s}-l(l+1) \dot{a}]=0, \\
\bar{\sigma} R^{-2} \hat{k} Y_{, \theta}+\delta p_{, \theta} R^{-2}=0 .
\end{gathered}
$$

The transition conditions (3.22) and (3.23) imply for $\lambda=0$ that $a^{\prime}$ and $s^{\prime}$ are continuous, as we expected. Therefore in Eq. (6.14) the undifferentiated $a$ and $s$ terms cancel for $\lambda$ $=0$ and we obtain

$$
\begin{aligned}
{\left[\left(\frac{1}{2}[2 \hat{k}]_{, r} Y\right)^{+}+\left(\frac{1}{2}[\hat{k}]_{, r} Y\right)^{-1}\right] \bar{\sigma} } \\
+\left(M^{+}+M^{-}\right) R^{-2}\left[\delta \sigma-\delta t_{00}(\bar{\sigma})\right] \\
+\left[\left(-\frac{1}{2} r^{2}\left[4 s_{, r}-2 l(l+1) a_{, r}\right]\right)^{+}\right. \\
\left.+\left(-\frac{1}{2} r^{2}\left[4 s_{, r}-2 l(l+1) a_{, r}\right]\right)^{-}\right] \bar{p} R^{-2} Y \\
-4 \delta p R^{-1}=0 .
\end{aligned}
$$

These equations contain the fields $a(t), s(t)$, which describe the motion of the shell, the matter fields $\delta \sigma, \delta p$, and the gravitational potential $\hat{k}$. We can solve the field equation in the case $\lambda=0$ to determine $\hat{k}$ in terms of the other variables (Appendix B):

$$
0=\delta R_{r \theta}=a^{\prime}-s^{\prime} .
$$

The $\theta \theta$ component of the field equations has terms proportional $Y$ and $Y_{, \theta \theta}$ which must vanish separately:

$$
\begin{gathered}
0=\delta R_{\theta \theta}=-r^{2}\left[s^{\prime \prime}+\frac{4 s^{\prime}}{r}-l(l+1) \frac{a^{\prime}}{r}+\frac{2 s}{r^{2}}\right]+l(l+1) s, \\
0=\delta R_{\theta \theta}=-r^{2}\left[a^{\prime \prime}+\frac{2 a^{\prime}}{r}+2 \frac{a}{r^{2}}\right]+2 a=-\left(r^{2} a^{\prime}\right)^{\prime} .
\end{gathered}
$$

Using $a^{\prime}=s^{\prime}, a^{\prime \prime}=s^{\prime \prime}$, we obtain from these equations the unique solution

$$
s=\frac{1}{r} g(t), \quad a=\frac{1}{r} g(t)+h(t) .
$$

We can simplify Eq. (6.17) if we use Eq. (6.21):

$$
\begin{aligned}
& \left({ }^{+} \hat{k}^{\prime}+{ }^{-} \hat{k}^{\prime}\right) \bar{\sigma}+\left(M^{+}+M^{-}\right) R^{-2} \delta \widetilde{\sigma}-s^{\prime}[4-2 l(l+1)] \bar{p} \\
& \quad-4 \delta p R^{-1}=0 .
\end{aligned}
$$

The field equation

$$
\begin{aligned}
0= & \delta R_{T r}=-\dot{s}^{\prime}-\frac{2}{r} \dot{s}+l(l+1) \dot{a}^{\prime}+l(l+1) \frac{1}{r} \dot{a}^{\prime} \\
& -l(l+1) \frac{1}{2 r}\left[\hat{\xi}^{\prime}+\frac{1}{r} \hat{\xi}\right]
\end{aligned}
$$

determines

$$
\hat{\xi}=\dot{h}\left(-\frac{R^{2}}{r}+r\right)
$$

where we have used that $\hat{\xi}$ vanishes at the shell. The $\delta R_{t \theta}$ $=0$ field equation is satisfied for such $s, a, \hat{\xi}$.

It remains to calculate $\hat{k}$ from $\delta R_{T T}$ :

$$
\begin{aligned}
0= & \delta R_{T T}=\hat{k}^{\prime \prime}+\frac{2}{r} \hat{k}^{\prime}-l(l+1) \frac{\hat{k}}{r^{2}}-2 \ddot{s}+l(l+1) \ddot{a} \\
& +\left(2 s^{\prime}-l(l+1) a^{\prime}\right) \bar{\Phi}^{\prime} \lambda^{-1}+l(l+1) \dot{h}\left(\frac{R^{2}}{r^{2}}-1\right) .
\end{aligned}
$$

If we insert $a, s, \hat{\xi}$, we obtain $\left(c^{2} \Phi^{\prime}=M / R^{2}\right)$

$$
\begin{aligned}
0= & \delta R_{T T}=\hat{k}^{\prime \prime}+\frac{2}{r} \hat{k}^{\prime}-l(l+1) \frac{1}{r^{2}} \hat{k} \\
& -[2-l(l+1)] \frac{1}{r} \ddot{g}-[2-l(l+1)] \frac{1}{r^{4}} M^{ \pm} g \\
& +l(l+1) \frac{R^{2}}{r^{2}} \ddot{h} .
\end{aligned}
$$

Because we are in the Gaussian gauge, we have to solve this equation on both sides of the shell with the prescribed jump of the normal derivatives at $R$ :

$$
{ }^{+} \hat{k}^{\prime}-{ }^{-} \hat{k}^{\prime}=4 \pi \delta \widetilde{\sigma}
$$

The solutions of the homogeneous equation are (regular at the origin and bounded at infinity)

$$
-\hat{k}_{0}=\alpha(t) \frac{r^{l}}{R^{l}}, \quad+\hat{k}_{0}=\beta(t) \frac{R^{l+1}}{r^{l+1}} .
$$

A particular solution of the inhomogeneous equation can be guessed: 


$$
{ }^{ \pm} \hat{k}_{p}=\ddot{g} r+g \frac{1}{r^{2}} M^{ \pm}+\ddot{h} R^{2} .
$$

Continuity at $R$ implies

$$
\alpha+g \frac{1}{R^{2}} M^{-}=\beta+g \frac{1}{R^{2}} M^{+} .
$$

The jump condition is

$$
-\beta(l+1) \frac{1}{R}-2 g \frac{1}{R^{3}} M^{+}-\alpha l \frac{1}{R}+2 g \frac{1}{R^{3}} M^{-}=4 \pi \delta \widetilde{\sigma} .
$$

From this we obtain

$$
\begin{gathered}
\beta=-\frac{l+2}{2 l+1} g \frac{1}{R^{2}}\left(M^{+}-M^{-}\right)-\frac{1}{2 l+1} 4 \pi R \delta \widetilde{\sigma}, \\
\alpha=\frac{l-1}{2 l+1} g \frac{1}{R^{2}}\left(M^{+}-M^{-}\right)-\frac{1}{2 l+1} 4 \pi R \delta \widetilde{\sigma} .
\end{gathered}
$$

Hence at $R$, the location of the shell, we obtain

$$
\begin{aligned}
-\hat{k}= & \frac{l-1}{2 l+1} g \frac{1}{R^{2}}\left(M^{+}-M^{-}\right)-\frac{1}{2 l+1} 4 \pi R \delta \widetilde{\sigma}+\ddot{g} R \\
& +g \frac{1}{R^{2}} M^{-}+\ddot{h} R^{2}, \\
+\hat{k}=- & \frac{l+2}{2 l+1} g \frac{1}{R^{2}}\left(M^{+}-M^{-}\right)-\frac{1}{2 l+1} 4 \pi R \delta \widetilde{\sigma}+\ddot{g} R \\
& +g \frac{1}{R^{2}} M^{+}+\ddot{h} R^{2},
\end{aligned}
$$

or because of $\ddot{g} R+\ddot{h} R^{2}=\ddot{a} R^{2}$,

$$
\begin{aligned}
\hat{k}= & -\hat{k}={ }^{+} \hat{k}=\ddot{a} R^{2}+\left[\frac{l+1}{2 l+1} M^{-}+\frac{l-1}{2 l+1} M^{+}\right] \frac{1}{R^{2}} g \\
& -\frac{1}{2 l+1} 4 \pi R \delta \widetilde{\sigma} .
\end{aligned}
$$

Finally, we need the derivatives

$$
\begin{aligned}
-\hat{k}^{\prime}= & l \frac{l-1}{2 l+1} g \frac{1}{R^{3}}\left(M^{+}-M^{-}\right)-\frac{l}{2 l+1} 4 \pi \delta \widetilde{\sigma} \\
& +\ddot{g}-2 g \frac{1}{R^{3}} M^{-} \\
+\hat{k}^{\prime}= & (l+1) \frac{l+2}{2 l+1} g \frac{1}{R^{3}}\left(M^{+}-M^{-}\right)+\frac{l+1}{2 l+1} 4 \pi \delta \widetilde{\sigma} \\
+ & \ddot{g}-2 g \frac{1}{R^{3}} M^{+}
\end{aligned}
$$

In the equation for the radial motion, we need

$$
\begin{aligned}
-\hat{k}^{\prime}+{ }^{+} \hat{k}^{\prime}= & 2 \ddot{g}+\frac{2 l(l-1)}{2 l+1} \frac{M^{+}}{R^{3}} g \\
& -\frac{2(l+1)(l+2)}{2 l+1} \frac{M^{-}}{R^{3}} g+\frac{4 \pi \delta \widetilde{\sigma}}{2 l+1} .
\end{aligned}
$$

Now we insert Eq. (6.35) into Eq. (6.16) for the tangential motion $(\delta p=\delta \tilde{p} Y)$

$$
\begin{gathered}
\bar{\sigma}\left(\ddot{g} R+\ddot{h} R^{2}+\left[\frac{l+1}{2 l+1} M^{-}+\frac{l-1}{2 l+1} M^{+}\right] \frac{1}{R^{2}} g\right. \\
\left.-\frac{1}{2 l+1} 4 \pi R \delta \sigma\right)+\delta \tilde{p}=0 .
\end{gathered}
$$

Similarly, we put Eq. (6.38) into Eq. (6.22) for the radial motion:

$$
\begin{aligned}
& \left(2 \ddot{g}+\frac{2 l(l-1)}{2 l+1} \frac{M^{+}}{R^{3}} g-\frac{2(l+1)(l+2)}{2 l+1} \frac{M^{-}}{R^{3}} g+\frac{4 \pi}{2 l+1}\right. \\
& \quad+g \frac{2}{R^{2}}[2-l(l+1)] \bar{p}+\left[\left(M^{+}+M^{-}\right) R^{-2}\right. \\
& \left.-2 \alpha R^{-1}\right] \delta \widetilde{\sigma}=0 .
\end{aligned}
$$

We can replace the background pressure $\bar{p}$ in the radial equation by

$$
\frac{4 \bar{p}}{R}=\bar{\sigma} \frac{M^{+}-M^{-}}{R^{2}}
$$

We can integrate Eq. (6.15), the continuity equation, to

$$
\delta \widetilde{\sigma}+\bar{\sigma}[2 s-l(l+1) a]=0,
$$

because the value of $a(t)$ at some time is arbitrary. Thus we can eliminate $\delta \widetilde{\sigma}$ from Eqs. (6.39), (6.40) and obtain two linear second-order equations for $g(t)$ and $a(t)$. The coefficients are determined by $M^{+}, M^{-}$, and $\alpha$, which characterize the equation of state of the perturbed shell.

We write these equations explicitly for the case $M^{-}=0$ :

$$
\begin{aligned}
& \bar{\sigma}\left(R^{2} \ddot{a}+\frac{l-1}{2 l+1} M^{+} \frac{1}{R^{2}} g-\frac{1}{2 l+1} 4 \pi R \delta \sigma\right)+\delta \widetilde{p}=0 \\
& \left(2 \ddot{g}+\frac{2 l(l-1)}{2 l+1} \frac{M^{+}}{R^{3}} g+\frac{4 \pi \delta \widetilde{\sigma}}{2 l+1}\right) \bar{\sigma} \\
& \quad+g \frac{2}{R^{2}}[2-l(l+1)] \bar{p}+\left(M^{+} R^{-2}-2 \alpha R^{-1}\right) \delta \widetilde{\sigma}=0 .
\end{aligned}
$$

Inserting $\bar{p}$ and $\delta \widetilde{\sigma}$, we get 


$$
\begin{aligned}
& \ddot{a}+\frac{M^{+}}{R^{4}} {\left[\frac{l+1}{2 l+1}-\frac{2 \alpha R}{M^{+}}\right] g } \\
&+\frac{M^{+}}{R^{3}}\left[-\frac{l(l=1)}{2 l+1}+\frac{l(l+1) \alpha R}{M}\right] a=0
\end{aligned}
$$

and

$$
\begin{array}{r}
\ddot{g}+\frac{M^{+}}{R^{3}}\left[\frac{l^{2}-3 l-2}{2 l+1}+\frac{2-l(l+1)}{4}+\frac{4 \alpha R}{M^{+}}\right] g \\
+\frac{M^{+}}{R^{2}}\left[\frac{l(l+1)^{2}}{2 l+1}-\frac{2 l(l+1) \alpha R}{M^{+}}\right] a=0 .
\end{array}
$$

These two equations are Eqs. (86) and (87) in [11] if we put $\eta=a$ and $\xi=g$. They describe the Newtonian oscillations. As linear ordinary differential equations with constant coefficients, they can be integrated in closed form. In [11] it is shown that Newtonian nonradial oscillations are always unstable. This indicates that there should be relativistic normal modes because it seems unlikely that the damping by gravitational waves could overcompensate the exponential growth of the Newtonian mode.

\section{ACKNOWLEDGMENTS}

I want to thank Jürgen Ehlers for very helpful discussions on the theory of shells, the Newtonian limit, and his careful reading of the manuscript. Thanks also to Jiri Bičák for his help with the Newtonian equations. Alan Rendall, Karel Kuchař, and Petr Hájíč̌k made valuable suggestions.

\section{APPENDIX A: THE NEWTONIAN SHELL}

In Sec. II a fluid shell was defined in the frame theory. I want to show that this way we really obtain a shell in New- tonian theory. We assume that the reader is familiar with the frame theory [9].

The conditions of continuity of the time and space metrics at $\Sigma$ imply that $\Sigma$ is a smooth hypersurface in the usual $\left(t, x^{i}\right)$ coordinates of Newtonian theory. From Eqs. (2.4), (2.5) we conclude $\Delta k_{\alpha \beta}=4 \pi \sigma t_{\alpha} t_{\beta}$. Hence the second-fundamental forms of $\Sigma_{t}$, the intersection of $\Sigma$ with the Newtonian time slices, coincide from both sides. Suppose that $x^{i}\left(t, a^{B}\right)$ are the integral curves of the fluid flow which $\operatorname{span} \Sigma$. Then $u^{\alpha} \partial_{\alpha}=\partial_{t}+\dot{x}^{i} \partial_{i}$ is the fluid flow vector. The definition of the second-fundamental form implies

$$
{ }^{+} k_{\alpha \beta} u^{\alpha} u^{\beta}=u{ }_{\alpha}^{; \beta} u^{\beta} n_{\alpha}=\left(\ddot{x}^{i}+\Phi^{, i}\right) n_{i},
$$

where we used the form of the Newtonian connection in standard coordinates:

$$
\Gamma_{\gamma \delta}^{\alpha}=s^{\alpha \beta} \Phi_{, \beta} t_{\gamma \delta}
$$

Relation (2.7) is

$$
\sigma\left({ }^{+} k_{\alpha \beta}+{ }^{-} k_{\alpha \beta}\right) u^{\alpha} u^{\beta}+\left({ }^{+} k_{\alpha \beta}+{ }^{-} k_{\alpha \beta}\right) p\left(s^{\alpha \beta}-n_{\bullet}^{\alpha} n_{\bullet}^{\beta}\right)=0
$$

or

$$
\sigma\left(\left(\ddot{x}^{i}+{ }^{+} \Phi^{, i}\right) n_{i}+\left(\ddot{x}^{i}+{ }^{-} \Phi, i\right) n_{i}\right)+2 \theta p=0,
$$

where $\theta$ is the trace of the second-fundamental form of $\Sigma_{t}$. This is what we would expect for the normal component of the equation of motion.

Similarly, the component of $\tau^{\alpha \beta}{ }_{\mid \beta}$ in the direction of $u^{\alpha}$ contains the mass conservation and the component in $\Sigma_{t}$ the tangential force. Finally, Eq. (2.3) determines the gravitational potential

$$
\Delta \Phi=4 \pi \sigma \delta_{\Sigma_{t}}
$$

\section{APPENDIX B: THE LINEARIZED CONNECTION AND RICCI TENSOR}

From the thesis of Kind [12], we have the linearized connection

$$
\begin{aligned}
& \delta \Gamma_{T T}^{T}=\left[\dot{k}+c e^{\Phi-\Lambda} \Phi^{\prime} \eta\right] Y, \\
& \delta \Gamma_{T r}^{T}=k^{\prime} Y, \\
& \delta \Gamma^{r}{ }_{T T}=c^{2} e^{2(\Phi-\Lambda)}\left[k^{\prime}+2 \Phi^{\prime}(k-\mu)\right] Y+c e^{\Phi-\Lambda} \dot{\eta} Y, \\
& \delta \Gamma_{r T}^{r}=\left[\dot{\mu}-c e^{\Phi-\Lambda} \Phi^{\prime} \eta\right] Y, \\
& \delta \Gamma_{r r}^{T}=c^{-2} e^{2(\Phi-\Lambda)} \dot{\mu} Y-c^{-1} e^{\Phi-\Lambda}\left(\eta^{\prime}+\Phi^{\prime} \eta\right) Y, \\
& \delta \Gamma_{r r}^{r}=\mu^{\prime} Y, \\
& \delta \Gamma^{T}{ }_{T \theta}=k Y_{, \theta} \\
& \delta \Gamma^{\theta}{ }_{T T}=c^{2} r^{-2} e^{2 \Phi} k Y_{, \theta}+c r^{-1} e^{\Phi}\left[\xi-c e^{\Phi-\Lambda} \Phi^{\prime} \omega\right] Y_{, \theta}, \\
& \delta \Gamma_{\theta T}^{\theta}=\dot{s} Y+\dot{a} Y_{, \theta \theta}, \\
& \delta \Gamma_{\theta \theta}^{T}=r^{2} c^{-2} e^{-2 \Phi}\left[\dot{s} Y+\dot{a} Y_{, \theta \theta}\right]-r c^{-1} e^{-\Phi}\left[e^{-\Lambda} \eta Y+\xi Y, \theta \theta\right],
\end{aligned}
$$


$\delta \Gamma_{T \phi}^{T}=0$,

$\delta \Gamma_{T T}^{\phi}=0$,

$\delta \Gamma_{\phi T}^{\phi}=\dot{s} Y+\dot{a} \cot \theta Y{ }_{, \theta}$,

$\delta \Gamma_{\phi \phi}^{T}=r^{2} c^{-2} e^{-2 \Phi} \sin ^{2} \theta\left[\dot{s} Y+\dot{a} \cot \theta Y_{, \theta}\right]-r c^{-1} e^{-\Phi} \sin ^{2} \theta\left[e^{-\Lambda} \eta Y+\xi \cot \theta Y_{, \theta}\right]$,

$\delta \Gamma_{r \theta}^{r}=\left[\mu-e^{-\Lambda} \omega\right] Y_{, \theta}$,

$\theta \Gamma^{\theta}{ }_{r r}=-r^{-2} e^{2 \Lambda} \mu Y_{, \theta}+r^{-1} e^{\Lambda}\left[\omega^{\prime}+r^{-1} \omega\right] Y_{, \theta}$,

$\delta \Gamma_{\theta r}^{\theta}=s^{\prime} Y+a^{\prime} Y_{, \theta \theta}$,

$\delta \Gamma_{\theta \theta}^{r}=-r^{2} e^{-2 \Lambda}\left[\left(s^{\prime}+2 r^{-1} s\right) Y+\left(a^{\prime}+2 r^{-1} a\right) Y_{, \theta \theta}\right]+r e^{-\Lambda}\left[e^{-\Lambda} 2 \mu Y+\omega Y_{, \theta \theta}\right]$,

$\delta \Gamma^{r}{ }_{r \phi}=0$,

$\delta \Gamma_{r r}^{\phi}=0$

$\delta \Gamma_{\phi r}^{\phi}=s^{\prime} Y+a^{\prime} \cot \theta Y_{, \theta}$

$\delta \Gamma_{\phi \phi}^{r}=-r^{2} e^{-2 \Lambda} \sin ^{2} \theta\left[\left(s^{\prime}+2 r^{-1} s\right) Y+\left(a^{\prime}+2 r^{-1} a\right) \cot \theta Y_{, \theta}\right]+r e^{-\Lambda} \sin ^{2} \theta\left[e^{-\Lambda} 2 \mu Y+\omega \cot \theta Y_{, \theta}\right]$,

$\delta \Gamma_{r \theta}^{T}=-(2 c)^{-1} e^{\Lambda-\Phi} \eta Y_{, \theta}-r(2 c)^{-1} e^{-\Phi}\left[\xi^{\prime}+\xi\left(\Phi^{\prime}-r^{-1}\right)-c^{-1} e^{\Lambda-\Phi} \dot{\omega}\right] Y_{, \theta}$,

$\delta \Gamma_{T \theta}^{r}=\frac{1}{2} c e^{\Lambda-\Phi} \eta Y_{, \theta}-\frac{1}{2} r c e^{\Phi-2 \lambda}\left[\xi^{\prime}+\xi\left(\Phi^{\prime}+r^{-1}\right)-c^{-1} e^{\Lambda-\Phi} \dot{\omega}\right] Y_{, \theta}$,

$\delta \Gamma_{T r}^{\theta}=-\frac{1}{2} c r^{-2} e^{\Lambda+\Phi} \eta Y_{, \theta}+c(2 r)^{-1} e^{\Phi}\left[\xi^{\prime}+\xi\left(r^{-1}-\Phi^{\prime}\right)+c^{-1} e^{\Lambda-\Phi} \dot{\omega}\right] Y_{, \theta}$,

and the linearized Ricci tensor

$$
\begin{aligned}
& \delta R_{T T}=\left\{-\ddot{\mu}-2 \ddot{s}+l(l+1) \ddot{a}+c e^{\Phi-\Lambda}\left[\dot{\eta}^{\prime}+\dot{\eta}\left(\Phi^{\prime}+2 r^{-1}\right)\right]-c e^{\Phi} r^{-1} l(l+1)\left[\xi-c e^{\Phi-\Lambda} \Phi^{\prime} \omega\right]\right. \\
& +c^{2} e^{2(\Phi-\Lambda)}\left[k^{\prime \prime}+k^{\prime}\left(2 \Phi^{\prime}-\Lambda^{\prime}+2 r^{-1}\right)+2 s^{\prime} \Phi^{\prime}-\mu^{\prime} \phi^{\prime}-l(l+1) a^{\prime} \Phi^{\prime} 2(k-\mu)\left(\Phi^{\prime \prime}+\Phi^{\prime} \Phi^{\prime}-\Phi^{\prime} \Lambda^{\prime}+2 r^{-1} \Phi^{\prime}\right)\right] \\
& \left.-c^{2} e^{2 \Phi} r^{-2} l(l+1) k\right\} Y, \\
& \delta R_{T r}=\left\{-2 \dot{s}^{\prime}+2 \dot{s}\left(\Phi^{\prime}-r^{-1}\right)+2 r^{-1} \dot{\mu}+l(l+1) \dot{a}^{\prime}-l(l+1) \dot{a}\left(\Phi^{\prime}-r^{-1}\right)\right. \\
& \left.-c e^{\Phi-\Lambda} \eta\left(\Phi^{\prime \prime}+\Phi^{\prime} \Phi^{\prime}-\Phi^{\prime} \Lambda^{\prime}+2 r^{-1} \Phi^{\prime}\right)-\frac{1}{2} c e^{\Phi} r^{-1} l(l+1)\left[\xi^{\prime}+\xi\left(r^{-1}-\Phi^{\prime}\right) c^{-1} e^{\Lambda-\Phi} \dot{\omega}-r^{-1} e^{\Lambda} \eta\right]\right\} Y \\
& \delta R_{r r}=\left\{c^{-2} e^{2(\Lambda-\Phi)} \ddot{\mu}-c^{-1} e^{\Lambda-\Phi}\left(\dot{\eta}^{\prime}+\Phi^{\prime} \dot{\eta}\right)-k^{\prime \prime}-2 s^{\prime \prime}+l(l+1) a^{\prime \prime}+k^{\prime}\left(\Lambda^{\prime}-2 \Phi^{\prime}\right)\right. \\
& \left.+\mu^{\prime}\left(\Phi^{\prime}+2 r^{-1}\right)-l(l+1)\left(\Lambda^{\prime}-2 r^{-1}\right)-e^{-\Lambda} r^{-1} l(l+1)\left(\omega^{\prime}+r^{-1} \omega\right)+e^{2 \Lambda} r^{-1} l(l+1) \mu\right\} Y, \\
& \delta R_{T \theta}=\left\{\dot{a}-\dot{\mu}-\dot{s}+\frac{1}{2} e^{-\Lambda} r\left[\dot{\omega}^{\prime}+\dot{\omega}\left(3 r^{-1}-g F^{\prime}\right)\right]+\frac{1}{2} c e^{\Phi-\Lambda} r\left[\xi^{\prime \prime}+\xi^{\prime}\left(\phi^{\prime}-\Lambda^{\prime}+2 r^{-1}\right)\right.\right. \\
& \left.+\xi\left(\Phi^{\prime \prime}-\Phi^{\prime} \Lambda^{\prime}+5 r^{-1} \Phi^{\prime}-r^{-1} \Lambda^{\prime}\right]\right\} Y_{, \theta},
\end{aligned}
$$

$\delta R_{r \theta}=\left\{a^{\prime}-k^{\prime}-s^{\prime}-k\left(\Phi^{\prime}-r^{-1}\right)+r(2 c)^{-1} e^{-\Phi}\left[-\dot{\xi}^{\prime}+\dot{\xi}\left(r^{-1}-\Phi^{\prime}\right)+c^{-1} e^{\Lambda-\Phi} \ddot{\omega}+e^{\Lambda} r^{-1} \dot{\eta}\right]+e^{-\Lambda} \omega\left(\Lambda^{\prime}-\Phi^{\prime}-r^{-1}\right)\right\} Y_{, \theta}$, 


$$
\begin{aligned}
\delta R_{\theta \theta}= & \left(c^{-2} e^{-2 \Phi} r^{2} \ddot{s}-c^{-1} e^{-\Phi} r \dot{\eta}-e^{-2 \Lambda} r^{2}\left\{s^{\prime \prime}+s^{\prime}\left(\Phi^{\prime}-\Lambda^{\prime}+4 r^{-1}\right)+r^{-1}\left[k^{\prime}-\mu^{\prime}-l(l+1) a^{\prime}\right]\right.\right. \\
& \left.\left.+(s-\mu) 2 r^{-1}\left(\phi^{\prime}-\Lambda^{\prime}+r^{-1}\right)\right\}+l(l+1)\left(s-e^{-\Lambda} \omega\right)\right) Y \\
& \times\left\{c^{-2} e^{-2 \Phi} r^{2} \ddot{a}-c^{-1} e^{-\Phi} r \dot{\xi}-e^{-2 \Lambda} r^{2}\left[a^{\prime \prime}+a^{\prime}\left(\Phi^{\prime}-\Lambda^{\prime}+2 r^{-1}\right)+a 2 r^{-1}\left(\Phi^{\prime}-\Lambda^{\prime}+r^{-1}\right)\right]\right. \\
& \left.+2 a-k-\mu+e^{-\Lambda} r\left[\omega^{\prime}+\omega\left(\Phi^{\prime}+r^{-1}\right)\right]\right\} Y_{\theta \theta}, \\
& \delta R_{\phi \phi}=\{\cdots\} \sin ^{2} \theta Y+\{\cdots\} \sin \theta \cos \theta Y_{, \theta} .
\end{aligned}
$$

In the brackets $\{\cdots\}$ the same expressions appear as in $\delta R_{\theta \theta}$.

\section{APPENDIX C: GAUGE TRANSFORMATIONS}

A general gauge transformation of an even perturbation with $Y_{l}(\theta)$ angular behavior is determined by

$$
\begin{gathered}
f_{t}=-c e^{\Phi} \alpha Y, \\
f_{r}=e^{\Lambda} \beta Y, \\
f_{\theta}=r \gamma Y_{, \theta},
\end{gathered}
$$

where $\alpha, \beta$, and $\gamma$ depend on $T, r$. The metric perturbations (3.1) change according to

$$
\begin{gathered}
k=k^{0}-\frac{1}{c} e^{-\Phi} \dot{\alpha}-e^{-\Lambda} \Phi^{\prime} \beta, \\
\eta=\eta^{0}-\frac{1}{c} e^{-\Phi} \dot{\beta}+e^{-\Lambda}\left(\alpha^{\prime}-\Phi^{\prime} \alpha\right), \\
\xi=\xi^{0}-\frac{1}{c} e^{-\Phi} \dot{\gamma}+\frac{1}{r} \alpha,
\end{gathered}
$$

$$
\begin{gathered}
\mu=\mu^{0}-e^{-\Lambda} \beta^{\prime}, \\
\omega=\omega^{0}-e^{-\Lambda}\left(\gamma^{\prime}-\frac{1}{r} \gamma\right)-\frac{1}{r} \beta, \\
s=s^{0}-e^{-\Lambda} \frac{1}{r} \beta, \\
a=a^{0}-\frac{1}{r} \gamma .
\end{gathered}
$$

The Regge-Wheeler gauge is characterized by $\xi=\omega=a=0$, as a consequence of the field equations $k=-\mu$. The following gauge transformation leads from a Gauss gauge to the Regge-Wheeler gauge:

$$
\begin{gathered}
\gamma=r a_{G}, \\
\beta=-e^{-\Lambda} r^{2}\left(a_{G}\right)^{\prime}, \\
\alpha=-r \xi_{G}+\frac{1}{c} e^{-\Phi} r^{2} \dot{a}_{G} .
\end{gathered}
$$

[1] W. Israel, Nuovo Cimento B 44, 1 (1966).

[2] J. E. Chase, Nuovo Cimento B 67, 136 (1966).

[3] J. Frauendiener, C. Hoenselaers, and W. Konrad, Class. Quantum Grav. 7, 585 (1990).

[4] J. Frauendiener and C. J. Klein, J. Math. Phys. 36, 3632 (1995).

[5] P. Hájícěk, B. S. Kay, and K. Kuchař, Phys. Rev. D 46, 5439 (1992).

[6] P. R. Brady, J. Louko, and E. Poisson, Phys. Rev. D 44, 1891
(1991).

[7] A. Ishibashi and H. Ishibara, Phys. Rev. D 56, 3446 (1997).

[8] S. Kind, J. Ehlers, and B. G. Schmidt, Class. Quantum Grav. 10, 2137 (1993).

[9] J. Ehlers, Class. Quantum Grav. 14, A119 (1997).

[10] H.-P. Nollert and B. G. Schmidt, Phys. Rev. D 45, 2617 (1992).

[11] J. Bičák and B. G. Schmidt, Report No. AE1-086.

[12] S. Kind, Ph.D. thesis, LMU München, 1992. 